\title{
Poésie et illustration, sous la direction de Lise Sabourin
}

\section{Annalisa Bottacin}

\section{(2) OpenEdition}

1 Journals

\section{Edizione digitale}

URL: http://journals.openedition.org/studifrancesi/6672

DOI: $10.4000 /$ studifrancesi.6672

ISSN: 2421-5856

\section{Editore}

Rosenberg \& Sellier

\section{Edizione cartacea}

Data di pubblicazione: 1 septembre 2010

Paginazione: 378-380

ISSN: 0039-2944

\section{Notizia bibliografica digitale}

Annalisa Bottacin, «Poésie et illustration, sous la direction de Lise Sabourin», Studi Francesi [Online], 161 (LIV | II) | 2010, online dal 30 novembre 2015, consultato il 08 janvier 2021. URL: http:// journals.openedition.org/studifrancesi/6672 ; DOI: https://doi.org/10.4000/studifrancesi.6672

Questo documento è stato generato automaticamente il 8 janvier 2021.

\section{(c) (i) $\odot$}

Studi Francesi è distribuita con Licenza Creative Commons Attribuzione - Non commerciale - Non opere derivate 4.0 Internazionale. 


\title{
Poésie et illustration, sous la direction de Lise Sabourin
}

\author{
Annalisa Bottacin
}

\section{NOTIZIA}

AA. VV., Poésie et illustration, sous la direction de Lise SABOURIN, Presses Universitaires de Nancy, «Centre d'Étude des Milieux Littéraires», 2008, pp. 417, ill.

Nella «Préface» (pp. I-VII), contrassegnata da una riflessione approfondita ed esaustiva, Lise SABOURIN evidenzia quanto il libro illustrato abbia, nel corso dei secoli, suscitato perplessità e critiche, motivate dal fatto che esso veniva sovente collegato «à l'instruction populaire, à l'éducation enfantine» (p. I). In primo luogo, l'illustrazione sulla copertina rende subito riconoscibile il libro; lo colloca in una precisa collana, lo posiziona in un catalogo e lo rende partecipe dello stile dell'editore. La copertina fonda il libro. È lì che il libro prende forma, diventando da oggetto impreciso e privo di espressività, a oggetto di appartenenza. La copertina gli conferisce il volto: essa stessa è letteratura. Ancor più ciò è reperibile dall'iconografia all'interno del volume, sorprendente rivelazione, posizionata qua e là tra le pagine: il lettore si trova davanti a una vera galleria di quadri che attivano reazioni, pensieri, osservazioni sulla pagina scritta, oltre che sui tratti salienti del disegno. Questo è l'alto merito del volume curato, e altresì arricchito da un suo saggio, da L. Sabourin, che porta a evidenziare come molti capolavori di arte grafica abbiano spesso reinventato la storia e si siano prestati in modo determinante alla diffusione dell'opera stessa. In particolar modo in campo poetico, dati i forti legami tra arte e letteratura. Il critico sottolinea la funzionalità dell'illustrazione, che rinvia a un rapporto di sguardo tra lettore e testo, assommato alla percezione di quanto l'autore ha prodotto; tutto ciò diventa un tratto distintivo dell'edizione, pari alla messa in scena di un'opera lirica che, privata del décor, rimarrebbe allo stadio di mera lettura del libretto (e della partitura per gli addetti ai lavori), a scapito dell'immaginario. In effetti, tutto ciò che permette di produrre il sogno, di attivare percorsi diversi, provoca nel lettore un ampliamento di rotta: 
l'illustrazione di un testo, oltre a riattivare e arricchire la lettura stessa, lo invita a una "curiosa" aspirazione alla ricerca, fuori dal mero limite della pagina scritta. «L'illustration-conclude L. Sabourin-assure donc un relais profitable entre générations par la mise en place d'une chaîne de créativités» (p. VII).

2 I contributi presenti in questo corposo e pregevole volume, che spaziano dal Medio Evo al Novecento, sono tutti degni di nota e la funzione estetica dell'iconografia è analizzata in testi di poesia, prosa e spazi teatrali.

Olivier CATEL, nel suo intervento dal titolo L"Agro romano" de Chateaubriand illustré par Maxime Dethomas (pp.189-201), allude alla nuova edizione di Léon Pichon de La Campagne romaine, Lettre à M. de Fontanes, Cynthie di Chateaubriand del 1919, arrichita dalle gravures di Maxime Dethomas, già illustratore di testi di Claudel, Daudet e Anatole France, in cui vengono rievocati paesaggi che, se si allontanano dalle visioni di Poussin sulla campagna romana, ne evidenziano, invero, i grandi contrasti di spazi indefiniti, di ombre e luci, di rovine, tanto cari a Chateaubriand; è certo il contrasto tra testi «plein[s] de lumière et de contours fins et épurés» e luoghi e figure che vengono da Dethomas tramutati in un linguaggio pittorico di «ombres épaisses»; seppur siano proprio queste ultime a rivelare, «par contraste [...], dans ses profondeurs, le rapport aux ombres du passé, aux figures sombres et obscures de l'imagination et du fantasme, souvent fuyantes et toujours impossibles à fixer, éternellement mystérieuses» (p. 190).

Barbara T. COOPER analizza in La "Jeanne d'Arc" d'Alexandre Soumet et son frontispice (pp. 204-15) l'illustrazione del libretto teatrale dell'edizione originale della pièce di Soumet, rappresentata per la prima volta all'Odéon, il 14 marzo 1825. Sul frontespizio appare l'immagine dell'interprete principale, in costume di scena, Mlle Georges, pseudonimo di Marguerite-Joséphine Wiemer, disegnata da Antoine o da Nicolas-Eustache Maurin. L'eroina appare in piedi sui ceppi ardenti, innalza con orgoglio la bandiera francese in segno di estremo amor di patria. La grande nobiltà d'animo e l'ardente coraggio sono evidenziati dai versi, tratti dall'ultima scena del dramma: «Dieu te mit dans mes mains, je te rapporte à lui» (p. 204), che conferiscono alla scena «une valeur intemporelle et sublime, un sens allégorique» (p. 205). Bisogna altresì ricordare che, all'epoca, le pièces drammatiche, presentate nel libretto con il frontespizio illustrato, non erano certo numerose e, come ben sottolinea il critico, nel caso particolare di Jeanne d'Arc, l'illustrazione si impone allo sguardo, producendo sullo spettatore un singolare esempio di glorificazione del personaggio martire. Sta a indicare che la pulzella d'Orléans è già santificata, oltre che assurta ai più alti gradi dell'eroismo; è una gloria nazionale che ribadisce alla nazione francese il ruolo della «monarchie de droit divin» (p. 215) - Charles X sarà incoronato a Reims il 29 maggio. Di fatto, così proposta, l'illustrazione della pièce, al pari dello stesso dramma, assume una funzione di propaganda, posizionandosi quali «actes politiques complémentaires à un moment précis de l'histoire de France» (p. 215).

5 Il primo dei due articoli dedicati a Théophile Gautier, dal titolo Des vignettes romantiques à l'illustration selon Gautier (1832-1838), è firmato da Nicolas WANLIN (pp. 217-228), che prendendo spunto da «Les Vignettes romantiques, ouvrage capital pour l'histoire du romantisme en général et du livre illustré en particulier» (p.217) di Champfleury, evidenzia l'importanza data talvolta più all'illustratore che all'autore stesso, nel fenomeno dei libri illustrati; in effetti il libro si afferma sia per l'opera in sé che per il disegno, spesso accompagnato da una didascalia che decora e illustra la pagina. All'inizio degli anni '30, Gautier, alla ricerca di riconoscimenti in campo poetico, 
interpreta positivamente quella che sempre più si concretizza in forma di pubblicità per l'opera stessa: l'illustrazione appare «la formule éditoriale idéale à la réunion d'écrivains et d'artistes» (p. 218). Nel 1834, Gautier era stato incaricato di scrivere un poema «devant faire face à une vignette dans un keepsake, L'Églantine» (p. 218), fatto che ben evidenzia come lo scrittore si sia ben inserito in quell'ottica, e nella sua Histoire $d u$ Romantisme così si esprime: "C'était l'usage alors d'aller demander aux littérateurs ancore heureux d'être imprimés au bout de vers ou de prose pour servir de texte à ces splendides illustrations des Robinson, des Cousin, des Findey, des Westall et des Prout» (p. 229). Wanlin prosegue la sua indagine delineando una serie di esemplificazioni atte a evidenziare la relazione tra letteratura e arti grafiche che, in ultima analisi, non giunge a diventare una "transformation d'art»; per altro lo stesso Gautier, raffinatissimo scrittore e artista egli stesso, ha mirato all'arte come creatore e non quale «copiste servile», concetto da lui rifiutato categoricamente.

Utilizzando nel titolo del suo contributo un'esemplare espressione di Baudelaire, Marie-Hélène GIRARD in Gautier et l'illustration ou le "Dada de la beauté typographique" (pp. 229-243) esordisce presentanto un Gautier "artiste" che sovente si confronta con le illustrazioni librarie, vivendo egli in quel milieu di pittori e disegnatori «qui se faisaient aussi illustrateurs» (p. 229). Frequentemente Gautier è stato attratto da opere illustrate in raffinati volumi, come ad esempio dagli album di Garavani o dall'edizione di Hamlet con le litografie di Delacroix. Egli stesso prese parte a simili progetti, come nel 1845, per un'edizione del suo Voyage pittoresque en Algérie con vignette e planches, che comunque non uscì, e collaborò con Gustave Doré per le illustrazioni dell'edizione di Capitaine Fracasse del 1866. Fatto eccezionale a dire di Girard: non vi è opera quale Émaux et Camées, dalle magiche forme, che pare scritta per attrarre un alto numero di illustratori (come ben rilevò Madeleine Cottin nell'edizione dell'opera del 1968); raccolta poetica che non trovò quel tipo di consenso, rimanendo fuori da tale moda. Girard rivolge dunque la sua attenzione alla ricerca di precise motivazioni che forniscano un chiarimento su questo inspiegabile fatto, su cui organizza un discorso dettagliato e preciso - che sarebbe da seguire passo passo arricchendo la sua valutazione di tracce, approfondimenti, proponendo ipotesi e rivelando notizie. In ultima analisi la studiosa conclude che Gautier, grande interprete di sguardi sulla modernità, tralascia in Émaux et Camées l'illustrazione in quanto «le poème est déjà en lui-même un objet pour les yeux et [...] il ne laisse pas grande latitude à l'illustrateur» (p. 243).

7 Laurence RICHER ("La fin du monde est mon seul rêve» ou "La Légende du Juif errant" autour de Gustave Doré, pp. 245-59) analizza una delle più diffuse leggende del Medioevo che ha lasciato traccia in tutte le letterature europee, le cui prime versioni risalgono alla fine del xv secolo, nella Bibliothèque bleue, tanto da costituire, per l'appunto, un tema legato all'«imagerie populaire» (p. 246). In epoca romantica, nel 1831, Nerval traduce un poema di Schubart e simbolica è l'epopea in prosa dialogata, Ahasvérus, firmata da Edgar Quinet e pubblicata nel 1833, specie di moderno mystère o di sacra rappresentazione. L'edizione presa in esame da Richer è quella uscita nel 1856 per i tipi di Michel Lévy frères, dal titolo La Légende du Juif errant, «un livre in-folio combinant partition, poésie et gravures sur bois» (ibid.), il cui grande pregio è quello di essere stata illustrata da Gustave Doré, con una serie di dodici gravures che ben esaltano l'elaborata articolazione del testo: in effetti, nota il critico, Doré «utilise pleinement l'imaginaire né de la rencontre entre l'histoire et la légende» (p. 239). 
Valentina PONZETTO ("Namouna" et "Fortunio": des poèmes de Musset à l'opéra-comique, pp. 261-275) evidenzia quante volte la musica abbia illustrato i lavori poetici di Alfred de Musset e quanti compositori ne abbiano tratto ispirazione. Hippolyte Monpou fu il primo a comporre arie per L'Andalouse (1830) e per Venise (1831), contribuendo non poco ad assicurare il grande successo di questi poemi presso un vasto pubblico; Monpou fu solo il primo, seguito da musicisti più celebri quali Offenbach, Massenet, Lalo e Bizet. Se da un lato furono prodotte brevi romanze per voce e piano, di cui si hanno varie versioni (vengono qui presentate anche alcune partiture), due sono comunque le opere che si distinguono per singolarità e innovazione: La Chanson de Fortunio, poema inserito nella pièce Le Chandelier, e Namouna, sources per balletti e opéras-comiques. Pare che la trasposizione in musica de La Chanson de Fortunio d'Offenbach sia nata nelle coulisses della Comédie-Française, sotto lo sguardo dello stesso Musset che curava la messa in scena de Le Chandelier: di qui l'inizio del sodalizio tra i due artisti. Come rileva V. Ponzetto, di tutt'altra specie fu invece il rapporto tra Bizet e Musset, più distaccato e indipendente: Georges Bizet produrrà Djamileh, opéra-comique in un atto su libretto di Louis Gallet, tratto da Namouna (che servirà ugualmente da soggetto per l'omonimo balletto di Édouard Lalo), che sarà presentato all'Opéra-Comique nel maggio del 1872. «Si Djamileh s'inspire de Namouna, - scrive V. Ponzetto - en effet, elle prend néanmoins son libre essor d'œuvre nouvelle et originale, sans doute plus qu'il n'arrive d'habitude avec les transpositions lyriques d'œuvres littéraires» (p. 269).

9 In questo rilevante studio (Bellenger illustrateur des "Destinées": une lecture de Vigny en 1898, pp. 293-308), Lise SABOURIN conduce un'analisi che prende spunto dal primo centenario della nascita di Vigny, il 28 marzo 1897, giorno in cui alla ComédieFrançaise, Sylvain lesse il sonetto di Sully Prudhomme, À Alfred de Vigny, la cui edizione illustrata venne distribuita ai membri dell'Académie Française, con planches di BelleryDesfontaines et Dunki, e un frontespizio firmato da Georges Bellenger, litografo de «L'Artiste», pittore assai noto, che aveva esposto annualmente al Salon dal 1864 al 1895 , come pure a Londra e a Boston. Tra l'altro il fratello Clément aveva recentemente inciso i disegni di Louis Dunki per l'edizione Pelletan di Servitude et Grandeur militaires. Ora, non stupisce che Georges Bellenger venga scelto quale illustratore per Les Destinées che saranno edite da Pelletan nel 1898: "Édition officielle en quelque sorte,-rileva L. Sabourin-pour célébrer un poète romantique un peu oublié lors de la parution posthume de son recueil majeur en 1864, mais que l'esprit symboliste a souvent reconnu pour maître et que les problèmes religieux et militaires de cette "Belle Époque” invitent à relire» (p. 294). È un'edizione celebrativa che inserisce il poema Moïse del 1823 (opera giovanile pubblicata nella prima edizione del 1826 dei Poèmes antiques et modernes) accanto alle Destinées; si tratta di un elegante volume, molto pregiato, uscito in edizione numerata e limitata, con grande ricchezza d'immagini per illustrare $\mathrm{i}$ testi e che non solo permettono di intendere come Bellenger interpreti l'opera di Vigny, bensì quanto venga investito dalle emozioni percepite dalla lettura dei versi e come gestisca l'operazione di svelamento degli stessi, attraverso il disegno. Il suo tratto conduce infatti il lettore a una nuova significativa appropriazione dell'opera poetica di Vigny. Altresì rilevante notare come l'illustratore si ponga davanti a quei poemi ispirati a fonti bilbiche o evangeliche: è il caso di Moïse o del Mont des Oliviers; troviamo ancora tracce d'orientalismo in La Colère de Samson e d'esotismo in Wanda. Tipologie pittoriche che portano la studiosa a notare che «nous sommes donc confrontés à une illustration de qualité variable» (p. 301), produttrice di un autentico legame tra disegnatore e autore, e che ben evidenzia l'animo tormentato del poète- 
philosophe. È un'edizione, quella delle Destinées uscita in occasione del centenario della nascita di Vigny, che offre invero una serie di sorprese, in primo luogo l'ottima qualità tecnica della pubblicazione, atta a «corrobor[er] le sonnet officiel de Sully Prudhomme qui fait de Vigny un poète de l'idéal...» (p. 308). 